\title{
INDUCTIVELY PERFECT MAPS AND TRI-QUOTIENT MAPS
}

\author{
E. MICHAEL ${ }^{1}$
}

\begin{abstract}
It is proved that every tri-quotient map $f: X \rightarrow Y$ from a metric space $X$ onto a countable regular space $Y$, with each $f^{-1}(y)$ completely metrizable, is inductively perfect. It is not known to what extent all the hypotheses in this result are necessary, and that leads to some open questions regarding simple compactness properties of mappings between separable metric spaces.
\end{abstract}

1. Introduction. The purpose of this note is to give a partial answer to a question asked in [4], and to raise some related questions.

Our starting point is the following result from [2], where a map $\operatorname{map}^{2} X \rightarrow Y$ is called inductively perfect if there exists an $X^{\prime} \subset X$ such that $f\left(X^{\prime}\right)=Y$ and $f \mid X^{\prime}$ is perfect. $^{3}$

THeOREM 1.1 [2 Corollary 1.2]. If $f: X \rightarrow Y$ is an open map from a metric space $X$ onto a paracompact space $Y$, and if each $f^{-1}(y)$ is complete (with the given metric on $X),{ }^{4}$ then $f$ is inductively perfect.

Our next result (Theorem 1.3 below) deals with tri-quotient maps, which were introduced in [4]. The definition of these maps, which is slightly complicated, will be given in \$2. At this point, let us merely record that each of the following properties of a map $f: X \rightarrow Y$ implies that it is tri-quotient (see [4, Theorem 6.5]).

$1.2($ a) $f$ is open.

$1.2($ b) $f$ is inductively perfect.

1.2(c) $X$ is regular, $Y$ is first-countable and Hausdorff, every $f^{-1}(y)$ is Lindelöf, and $f$ is countable-compact-covering.

Concerning the terminology in 1.2(c), we call a map $f: X \rightarrow Y$ compact-covering (resp. countable-compact-covering) if every compact (resp. countable, compact) $K \subset Y$ is the image of some compact $C \subset X$. Since perfect maps have the property that the inverse image of every compact set is compact, it follows that every inductively perfect map is compact-covering (and hence surely countablecompact-covering). ${ }^{5}$

Received by the editors April 28, 1980 and, in revised form, July 14, 1980.

1980 Mathematics Subject Classification. Primary 54C10, 54C65, 54D30, 54E50.

Key words and phrases. Inductively perfect, tri-quotient, compact-covering, countable-compactcovering.

'Supported by an Alexander von Humboldt Foundation grant at the University of Stuttgart and by a National Science Foundation grant at the University of Washington.

${ }^{2}$ All maps in this paper are continuous and onto.

${ }^{3}$ This $X^{\prime}$ must be closed in $X$ if $X$ is Hausdorff; see, for instance, [3, Corollary 1.5].

${ }^{4}$ Example 5.1 shows that it does not suffice for each $f^{-1}(y)$ to be completely metrizable.

${ }^{5}$ By Theorem 3.1, an open map from a first-countable space onto a regular space must also be countable-compact-covering. 
The following result shows that, under fairly mild restrictions, the implication indicated by $1.2(\mathrm{~b})$ above is reversible.

TheOREM 1.3 ([4, THEOREM 6.6]). Every tri-quotient map $f: X \rightarrow Y$ from a complete metric space $X$ onto a paracompact space $Y$ is inductively perfect.

It was asked in [4, Question 1.9] whether Theorem 1.3 remains true if the assumption that $X$ is complete is weakened to assuming only that each $f^{-1}(y)$ is complete. (In view of 1.2(a), a positive answer would yield a simultaneous generalization of Theorems 1.1 and 1.3.) While the above question remains open in general, the following theorem, whose proof will be given in \$4, supplies a positive answer in case $Y$ is countable.

THEOREM 1.4. Every tri-quotient map $f: X \rightarrow Y$ from a metric space $X$ onto a countable regular space $Y$, with each $f^{-1}(y)$ completely metrizable, is inductively perfect.

We now ask another question: Does Theorem 1.4 remain true without assuming that each $f^{-1}(y)$ is completely metrizable? It should be remarked that the answer is "yes" if $f$ is open, for then $f$ actually maps some $X^{\prime} \subset X$ homeomorphically onto $Y$ (see Theorem 3.1). This should be contrasted to Example 5.1, where $f$ is open but $Y$ is not countable.

Theorems 1.3 and 1.4, as well as the questions following them, retain their interest if the assumption that $f$ is tri-quotient is strengthened to assuming that $f$ has property $1.2(\mathrm{c})$. In the case of Theorem 1.3, one then obtains a result of $\mathrm{A}$. V. Ostrovskii [6, Theorem 1]. In the case of the questions, one obtains-after further strengthening and simplifying the hypotheses-the following formulations.

Question 1.5. Suppose $f: X \rightarrow Y$ is a map from a separable metric space $X$ onto a metric space $Y$, with each $f^{-1}(y)$ compact. If $f$ is countable-compact-covering, must $f$ be compact-covering? And if $f$ is compact-covering, must $f$ be inductively perfect?

QUESTION 1.6. Suppose $f: X \rightarrow Y$ is a compact-covering map from a separable metric space $X$ onto a countable metric space $Y$. Must $f$ be inductively perfect?

Observe that Questions 1.5 and 1.6 deal only with simple compactness properties of mappings between separable metric spaces. We conclude this introduction with some further remarks about these questions.

(1.7). I do not know the answer to the first part of Question 1.5 even when $Y=I$ (closed interval), $X \subset Y \times I$, and $f(y, t)=y$.

(1.8). I do not know the answer to Question 1.6 even when $Y=Q$ (rationals), $X \subset Y \times I$, and $f(y, t)=y$.

(1.9). The answer to Questions 1.5 and 1.6 is "yes" if $f$ is open; see Theorem 1.1 (for Question 1.5) and Theorem 3.1 (for Question 1.6).

(1.10). The answer to both parts of Question 1.5 becomes "no" if the fibers are not assumed compact (or at least complete), even when $f$ is open. For the first part that follows from Example 5.1, and for the second part it follows from [4, Example 9.7]. 
(1.11). Regarding Question 1.6, I do not even know whether $f$ must have the property (which is clearly possessed by all inductively perfect maps) that every $f^{-1}(y)$ has a compact subset $K$ such that $f(U)$ is a neighborhood of $y$ in $Y$ whenever $U$ is a neighborhood of $K$ in $X$.

$\S \S 2$ and 3 contain the definition of tri-quotient maps and some preliminary results. The proof of Theorem 1.4 is given in $\$ 4$, and $\$ 5$ contains an example.

2. A lemma on tri-quotient maps. We begin with the following definition (see [4, Definition 6.1]).

Definition 2.1. A map $f: X \rightarrow Y$ is tri-quotient if one can assign to every open $U \subset X$ an open $U^{*} \subset Y$ such that:

(a) $U^{*} \subset f(U)$.

(b) $X^{*}=Y$.

(c) $U_{1} \subset U_{2}$ implies $U_{1}^{*} \subset U_{2}^{*}$.

(d) If $y \in U^{*}$, and if $\mathscr{W}$ is a cover of $f^{-1}(y) \cap U$ by open subsets of $X$, then there exists a finite $\mathscr{F} \subset \mathscr{W}$ such that $y \in(\cup \mathscr{F})^{*}$.

In the following lemma, a sequence $\left(A_{n}\right)$ of subsets of $X$ is called an outer network at a set $K \subset X$ if $K=\bigcap_{n} A_{n}$ and every open $G \supset K$ contains some $A_{n}$.

LEMMA 2.2. Suppose that $f: X \rightarrow Y$ is a tri-quotient map with $X$ metrizable and $Y$ a $T_{1}$-space, that $U \subset X$ is open, that $y \in U^{*}$, and that $f^{-1}(y)$ is completely metrizable. Then there exists a decreasing sequence $\left(U_{n}\right)$ of open subsets of $U$ such that $y \in U_{n}^{*}$ for all $n$ and $\left(U_{n}\right)$ is an outer network at a nonempty compact subset $K$ of $f^{-1}(y)$.

Proof. Let $\rho$ be a compatible complete metric on $f^{-1}(y)$, and let $X$ be metrized by a metric $d$ which extends $\rho$ (see [1]).

By an easy induction, we can use Definition 2.1 to construct a sequence $\left(\mathscr{F}_{n}\right)$ of finite collections of open subsets of $U$ such that for all $n$ :

(a) If $S \in \mathscr{F}_{n}$, then diam $S<1 / n$ and $S \cap f^{-1}(y) \neq \varnothing$.

(b) If $S \in \mathscr{F}_{n+1}$, then $\bar{S} \subset T$ for some $T \in \mathscr{F}_{n}$.

(c) $y \in\left(\cup \mathscr{F}_{n}\right)^{*}$.

Let $U_{n}=\cup \mathscr{F}_{n}$, and let us check that $\left(U_{n}\right)$ satisfies our requirements.

Clearly $U_{n} \subset U, U_{n+1} \subset U_{n}$ and $y \in U_{n}^{*}$ for all $n$. Now let $K=\cap_{n} U_{n}=$ $\cap_{n} \bar{U}_{n}$. Then $K \subset f^{-1}(y)$ by (a); hence $K$ is totally bounded and complete, and thus compact. It remains to show that every open $G \supset K$ contains some $U_{n}$; since $U_{n} \neq \varnothing$ for all $n$ (because $y \in U_{n}^{*}$ ), this will also prove that $K \neq \varnothing$.

Let $G \supset K$ be open in $X$, and suppose that $G \nsupseteq U_{n}$ for all $n$. Let $\mathscr{F}_{n}^{\prime}=\left\{S \in \mathscr{F}_{n}\right.$ : $S \nsubseteq G\}$. Then each $\mathscr{F}_{n}^{\prime}$ is finite and nonempty, and if $S \in \mathscr{F}_{n+1}^{\prime}$, then $S \subset T$ for some $T \in \mathscr{F}_{n}^{\prime}$. By König's Infinity Lemma, one can choose $S_{n} \in \mathscr{F}_{n}^{\prime}$ such that $S_{n+1} \subset S_{n}$ for all $n$. By (a) and the completeness of $f^{-1}(y)$, there exists an $x \in \cap_{n}\left(\bar{S}_{n} \cap f^{-1}(y)\right)$. Clearly $x \in K$, so $x \in G$ and hence $S_{n} \subset G$ for some $n$. But that is impossible (since $S_{n} \in \mathscr{F}_{n}^{\prime}$ ), and our proof is complete.

3. Some results on l.s.c. and u.s.c. maps. Recall that a function $\phi: Y \rightarrow 2^{Z}$ (where $\left.2^{Z}=\{A \subset Z: A \neq \varnothing\}\right)$ is l.s.c. (= lower semicontinuous) if $\{y \in Y: \phi(y) \cap V \neq$ $\varnothing\}$ is open in $Y$ for every open $V$ in $Z$. It is easy to see that a surjection $f: Z \rightarrow Y$ is 
open if and only if $f^{-1}: Y \rightarrow 2^{Z}$ is 1.s.c. The following result will be used in the proof of Theorem 1.4.

THEOREM 3.1 [5, THEOREM 1.1]. If $\phi: Y \rightarrow 2^{Z}$ is l.s.c., with $Y$ a countable ${ }^{6}$ regular space and $Z$ first-countable, then there exists a continuous $g: Y \rightarrow Z$ such that $g(y) \in \phi(y)$ for all $y \in Y$.

Now recall that a function $\phi: Y \rightarrow 2^{X}$ is u.s.c. (= upper semicontinuous) if $\{y \in Y: \phi(y) \subset V\}$ is open in $Y$ for every open $V$ in $X$. Since a surjection $f: X \rightarrow Y$ is closed if and only if the map $f^{-1}: Y \rightarrow 2^{X}$ is u.s.c., we are led to the following characterization.

Lemma 3.2. A continuous surjection $f: X \rightarrow Y$ is inductively perfect if and only if there exists an u.s.c. $\theta: Y \rightarrow 2^{X}$ such that $\theta(y)$ is a compact subset of $f^{-1}(y)$ for all $y \in Y$.

The following lemma will be applied in the proof of Theorem 1.4; for the definition of outer networks, see the paragraph preceding Lemma 2.2.

LEMMA 3.3. Let $X$ be a topological space. Let $\delta(X)=\prod_{n=1}^{\infty} \delta_{n}(X)$, where each $\mathcal{S}_{n}(X)$ is $2^{X}$ with the discrete topology. Let $\Re(X)$ be the set of all $S=\left(S_{n}\right)$ in $\delta(X)$ such that $\left(S_{n}\right)$ is an outer network at $\cap_{n} S_{n}$. Define $\psi: \Re(X) \rightarrow 2^{X}$ by $\psi(S)=$ $\cap_{n} S_{n}$. Then $\psi$ is u.s.c.

Proof. Let $S \in \Re(X)$, and suppose $\psi(S) \subset V$ with $V$ open in $X$. Then $S_{m} \subset V$ for some $m$. Let $\mathscr{W}=\left\{S^{\prime} \in \mathcal{R}(X): S_{m}^{\prime}=S_{m}\right\}$. Then $\mathscr{W}$ is a neighborhood of $S$ in $\Re(X)$ and $\psi\left(S^{\prime}\right) \subset V$ for all $S^{\prime} \in \mathcal{W}$. Hence $\psi$ is u.s.c. at $S$, and that completes the proof.

4. Proof of Theorem 1.4. Let $\Re(X)$ be as in Lemma 3.3, and let $Z \subset \Re(X)$ be the set of all $\left(U_{n}\right) \in \Re(X)$ such that $U_{n}$ is open in $X$ and $U_{n+1} \subset U_{n}$ for all $n$. For each $y \in Y$, let $\phi(y)$ be the set of $\left(U_{n}\right) \in Z$ such that $y \in U_{n}^{*}$ for all $n$ and $\left(U_{n}\right)$ is an outer network at some nonempty compact $K \subset f^{-1}(y)$. It follows from Lemma 2.2 (with $U=X$ ) that $\phi(y) \neq \varnothing$ for all $y \in Y$, so $\phi: Y \rightarrow 2^{Z}$.

Let us show that $\phi: Y \rightarrow 2^{Z}$ is l.s.c. We must show that, if $H \subset Z$ is open, then the set $W=\{y \in Y: \phi(y) \cap H \neq \varnothing\}$ is open in $Y$. It will suffice to prove this for every basic open $H \subset Z$ of the form

$$
H=\left\{\left(U_{n}\right) \in Z: U_{n}=V_{n} \text { for } n<n_{0}\right\},
$$

where $\left(V_{n}\right) \in Z$ and $n_{0} \in N$. We will prove that $W$ is open for this $H$ by showing that $W=V_{n_{0}}^{*}$.

That $W \subset V_{n_{0}}^{*}$ follows from the definitions. So suppose that $y \in V_{n_{0}}^{*}$, and let us check that $y \in W$. To do that, we must find some $\left(U_{n}\right) \in H \cap \phi(y)$, and that is easily done by letting $U_{n}=V_{n}$ for $n<n_{0}$ and then applying Lemma 2.2 (with $U=U_{n_{0}}$ ) to choose $U_{n}$ for $n>n_{0}$.

Having shown that $\phi: Y \rightarrow 2^{Z}$ is 1.s.c., we can apply Theorem 3.1 to obtain a continuous $g: Y \rightarrow Z$ such that $g(y) \in \phi(y)$ for all $y \in Y$. Now let $\psi: \Re(X) \rightarrow 2^{X}$

\footnotetext{
${ }^{6}$ The significance of $Y$ being countable is that it permits one to dispense with any completeness requirements on the sets $\phi(y)$.
} 
be as in Lemma 3.3, and define $\theta: Y \rightarrow 2^{X}$ by $\theta=\psi \circ g$. Since $\psi$ is u.s.c. (by Lemma 3.3), so is $\theta$. The definitions of $\phi, g$ and $\psi$ imply that $\theta(y)$ is a nonempty compact subset of $f^{-1}(y)$ for all $y \in Y$. Hence $f$ is inductively perfect by Lemma 3.2.

\section{An example.}

Example 5.1 [2, Example 4.1]. There exists an open map $f: X \rightarrow I$ from a separable metrizable space $X$ onto the closed interval $I$, with each $f^{-1}(y)$ completely metrizable, which is not compact-covering. (By Theorem 3.1, it is, however, countable-compact-covering.)

\section{REFERENCES}

1. F. Hausdorff, Erweiterung einer Homöomorphie, Fund. Math. 16 (1930), 353-360.

2. E. Michael, A theorem on semi-continuous set-valued functions, Duke Math. J. 26 (1959), 647-652.

3. __ A theorem on perfect maps, Proc. Amer. Math. Soc. 28 (1971), 633-634.

4. __ Complete spaces and tri-quotient maps, Illinois J. Math. 21 (1977), 716-733.

5. Continuous selections and countable sets, Fund. Math. (to appear).

6. A. V. Ostrovskii, On compact-covering mappings, Dokl. Akad. Nauk SSSR 227 (1976), 1297-1300; English transl. in Soviet Math. Dokl. 17 (1976), 606-610.

Department of Mathematics, University of Washington, Seattle, Washington 98195 